\title{
Diagnosis of rectal gonorrhoea by blind anorectal swabs compared with direct vision swabs taken via a proctoscope
}

\author{
P. DEHERAGODA \\ From the Department of Genito Urinary Medicine, Charing Cross Hospital, London W6
}

SUMMARY Eight hundred and twenty-three examinations were carried out on 662 homosexual patients. At each examination a blind anorectal swab and a rectal swab taken via a proctoscope were inoculated on to a culture plate. From a total of 100 gonococcal infections of the rectum 96 gave positive results from blind anorectal swabs and 99 from swabs taken via a proctoscope. Blind anorectal swabs proved to be a reliable method in the diagnosis of rectal gonorrhoea.

\section{Introduction}

In this study two methods for diagnosing rectal gonorrhoea were compared: the standard procedure of taking rectal swabs via a proctoscope and blind anorectal swabs. It was not intended to abandon proctoscopy but rather to assess the reliability of anorectal swabs in the diagnosis of gonorrhoea. Proctoscopy is not a pleasant procedure for the patient and women in particular may even refuse to have it done. The importance of examining the rectum for gonorrhoea is stressed by Schroeter and Reynolds (1972) who found that $65 \%$ of women with gonorrhoea in other sites had rectal involvement. Odegaard et al. (1971) showed that $53 \%$ of patients had rectal infections and Sparling et al. (1965) found rectal infection in $44 \%$ of women with gonorrhoea in other sites.

Anorectal cultures for Neisseria gonorrhoeae were taken on all female patients on their first visit and we found that $2 \%$ out of a total of 200 patients with positive results had a rectal infection only. Odegaard et al. (1971) found $2.7 \%$ and Bhattacharyya and Jephcott (1974) found that $5.6 \%$ had rectal infections only. The need to look for rectal infections in passive male homosexuals does not need to be stressed. A diagnostic technique which is reliable, easy to per-

Address for reprints: Dr P. Deheragoda, Department of Genito Urinary Medicine, Croydon General Hospital, London Road, Croydon, Surrey CR9 2RH

Received for publication 31 May 1977 form, and not distressing to the patient is needed, and this study was done with this in mind. It was carried out on male homosexuals as it was more convenient to study it in this type of patient.

\section{Method and material}

All homosexual men who admitted anal intercourse and who attended the clinic between April and November 1976 were studied. The anorectal swab was taken by inserting a sterile cotton wool swab about $1 \frac{1}{2}$ inches into the anal canal and this was inoculated into a divided blood agar plate which contained non-selective medium in one half and selective medium in the other half (Gibco Bio-Cult Diagnostics). The non-selective medium was inoculated first. The selective medium contained vancomycin $3 \mu \mathrm{g}$, nystatin 12.5 units, colistin $7.5 \mu \mathrm{g}$, and trimethoprim $5 \mu \mathrm{g} / \mathrm{ml}$. A proctoscope lubricated with K-Y Jelly was then passed and a second swab was taken by direct vision. The second swab was inoculated on to the same culture plate as shown in the Figure. One plate was used for both anorectal and proctoscopic swabs because this ensured that both cultures taken from the same patient were treated in an identical manner and incubated under the same conditions. The plate was then placed in a candle jar and incubated at $37^{\circ} \mathrm{C}$ for up to 72 hours. Growth of $N$. gonorrhoeae was confirmed by direct immunofluorescence. 


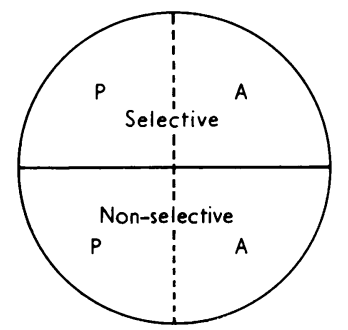

P -Proctoscopic swab (direct vision) A-Anorectal swab

Figure Diagram of culture plate illustrating inoculation procedure.

\section{Results}

Altogether 823 examinations were carried out on 662 patients and of the 823 culture plates thus obtained 100 showed a growth of $N$. gonorrhoeae. Of these 100 positive plates 95 were positive on both the anorectal and proctoscopic swabs. Of the remaining five positive plates one was positive on the anorectal swab but negative on the proctoscopic swab. The remaining four plates were positive on the proctoscopic swab but negative on the anorectal swab (Table 1). Ninety-four patients were diagnosed

Table 1 Diagnosis of gonorrhoea in 100 patients

\begin{tabular}{lll}
\hline Swab & & \\
\hline Anorectal & Proctoscopic & Both \\
\hline 96 & 99 & 100 \\
\hline
\end{tabular}

on the first visit and six on the second using either technique. Blind anorectal swabs gave a $91 \%$ isolation rate and proctoscopic swabs a $94 \%$ isolation rate on the first visit (Table 2). The diagnosis of

Table 2 Isolation rate on the first and second visits in 100 cases of gonorrhoea

\begin{tabular}{|c|c|c|c|}
\hline \multirow{2}{*}{$\begin{array}{l}\text { Positive } \\
\text { culture }\end{array}$} & \multicolumn{2}{|l|}{ Swab } & \multirow[b]{2}{*}{ Either (\%) } \\
\hline & Anorectal (\%) & Proctoscopic (\%) & \\
\hline \multicolumn{4}{|l|}{ Visit } \\
\hline First & 91 & 94 & 94 \\
\hline Second & 5 & 5 & 6 \\
\hline
\end{tabular}

rectal gonorrhoea from Gram-stained smears made from material obtained via a proctoscope has never been satisfactory, particularly in a busy clinic, and we found only $32 \%$ of smears with positive results.

Sixty-eight out of a total of 100 gonococcal rectal infections were asymptomatic and of the remaining
32 symptomatic infections 20 complained of a discomfort in the back or pruritus ani and 12 complained of a rectal discharge. Twenty-one had clinical proctitis (injection of the rectum and mucopus). It is interesting to compare these figures with those of 562 patients who did not have a gonococcal infection; $478(85 \%)$ were asymptomatic and of the remaining $84(15 \%), 33(6 \%)$ complained of either a discomfort in the back or pruritus ani and $51(9 \%)$ of a rectal discharge. Seventy-one $(12 \cdot 3 \%)$ had clinical proctitis (Table 3$)$.

Table 3 Symptoms and signs in 662 patients

\begin{tabular}{llllll}
\hline & \multicolumn{2}{l}{ Percentage and number of patients } \\
\cline { 2 - 3 } $\begin{array}{l}\text { Clinical } \\
\text { findings }\end{array}$ & \multicolumn{2}{l}{ Without } & gonorrhoea (562) & & \multicolumn{2}{l}{ With gonorrhoea (100) } \\
\cline { 2 - 3 } \cline { 5 - 6 } & No. & $\%$ & & No. & $\%$ \\
\hline No symptoms & 478 & $(85)$ & 68 & $(68)$ \\
Symptoms & 84 & $(15)$ & 32 & $(32)$ \\
Signs & 71 & $(12 \cdot 3)$ & 21 & $(21)$ \\
\hline
\end{tabular}

\section{Discussion}

The incidence of positive cultures obtained from blind anorectal swabs was not markedly different from that obtained using a proctoscope. This shows that a blind anorectal swab is a reliable method for the diagnosis of rectal gonorrhoea by culture. The diagnosis of rectal gonorrhoea from Gram-stained smears has never been very satisfactory, as shown by an isolation rate of only $32 \%$.

Blind anorectal swabs are now taken from all female patients attending this clinic and this has proved to be satisfactory for the detection of anorectal infections and more acceptable to the patients, particularly as women rarely complain of anorectal symptoms. This was our observation as well as that of Bhattacharyya and Jephcott (1974) who found that out of 32 women with positive results none complained of rectal symptoms.

Proctoscopy was in practice rarely of value in male homosexuals other than as a means of obtaining material for the diagnosis of gonorrhoea; primary and secondary syphilitic lesions were visible without the aid of a proctoscope, and warts in the anal canal always had accompanying perianal lesions. It is however, not suggested that a proctoscopic examination should not be done at all, but that it could be confined to the first visit and to patients who complain of symptoms. This comparative study has shown that an anorectal swab gives suitable material for the purpose of diagnosing rectal gonorrhoea by culture and for a test of cure after treatment. 
I should like to thank Dr B. A. Evans, and all my colleagues and the nursing staff of Luke Clinic for their valuable assistance.
References

Bhattacharyya, M. N., and Jephcott, A. E. (1974). Diagnosis of gonorrhoea in women: Role of the rectal sample. British Journal of Venereal Diseases, 50, 109-112.

Odegaard, K., Gundersen, H., and Gundersen, T. (1971). Rectumgonoré hos kvinner. Tidsskrift for den Norske Laegeforening, 91, 1474-1476.

Schroeter, A. L., and Reynolds, G. (1972). The rectal culture as a test of cure of gonorrhea in the female. Journal of Infectious Diseases, 125, 499-503.

Sparling, P. F., Yobs, A. R., Billings, T. E., and Hackney, J. F. (1965). Spectinomycin sulfate and aqueous procaine penicillin $G$ in treatment of female gonorrhea. Antimicrobial Agents and Chemotherapy, 5, 689-692. 